\title{
Statistical Hypo-Convergence in Sequences of Functions
}

\begin{abstract}
Şükrü Tortop
Department of Mathematics, Faculty of Art and Sciences, Afyon Kocatepe University, 03200 Afyonkarahisar, Turkey.
\end{abstract}

How to cite this paper: Tortop, S. (2018) Statistical Hypo-Convergence in Sequences of Functions. Journal of Applied Mathematics and Computation, 2(11), 504-512. DOI: $10.26855 /$ jamc.2018.11.002

*Corresponding author: Şükrü Tortop, Department of Mathematics, Faculty of Art and Sciences, Afyon Kocatepe University, 03200 Afyonkarahisar, Turkey. Email: stortop@aku.edu.tr

\section{Abstract}

In this paper, we define statistical hypo-convergence in metric spaces as an alternative to statistical pointwise and uniform statistical convergence. We show that this type of convergence provides a useful tool for solving stochastic optimization and variational problems. Also, its characterizations with level sets are obtained.

2010 AMS Classification: primary 40A30, secondary 40A35, 49J45.

\section{Keywords}

Statistical hypo-convergence, hypographs, upper semicontinuity, level sets.

\section{Introduction.}

Hypo-convergence focuses on hypo-graphs whereas epi-convergence deals with epigraphs. In literature, epi-convergence is more familiar than hypo-convergence and it is first studied by Wijsman [20,21] where it is called infimal convergence in the late of 1960's. After Wijsman's initial contributions, it is studied by Mosco [12] on variational inequalities, by Joly [8] on topological structures compatible with epi-convergence, by Salinetti and Wets [17] on equisemicontinuous families of convex functions, by Attouch [2] on the relationship between the epi-convergence of convex functions and the graphical convergence of their subgradient mappings, and by McLinden and Bergstrom [11] on the preservation of epi-convergence under various operations performed on convex functions. Furthermore, Dal Maso [10] called it $\Gamma$-convergence. The term epi-convergence is used by Wets [19] in 1980 for the first time. Epi-convergence is needed to solve some mathematical problems including stochastic optimization, variational problems and partial differential equations.

In this part fundamental definitions and theorems will be given. First of all, let $(X, d)$ be a metric space and $f,\left(f_{n}\right)$ are functions defined on $X$ with $n \in \mathbb{N}$. If it is not mentioned explicitly the symbol $d$ stands for the metric on $X$.

Let $K \subseteq \mathbb{N}$ and if the $\operatorname{limit} \delta(K)=\lim _{n} \frac{1}{n}|\{k \leq n: k \in K\}|$ exists then it is called asymptotic density of $K$ where $|\{k \leq n: k \in K\}|$ denotes the number of elements of $K$ not exceeding n (see[1, 13]).

$$
\begin{aligned}
& \text { If } \delta\left(K_{1}\right)=\delta\left(K_{2}\right)=1 \text {, then } \delta\left(K_{1} \cap K_{2}\right)=\delta\left(K_{1} \cup K_{2}\right)=1 . \\
& \text { If } \delta\left(K_{1}\right)=\delta\left(K_{2}\right)=0 \text {, then } \delta\left(K_{1} \cap K_{2}\right)=\delta\left(K_{1} \cup K_{2}\right)=0 .
\end{aligned}
$$

Statistical convergence of a sequence of scalars was introduced by Fast [5]. Let $x=\left(x_{k}\right)$ be a sequence of real or complex numbers. If for all $\varepsilon>0$, there exists $L$ such that,

$$
\lim _{n} \frac{1}{n}\left|\left\{k \leq n:\left|x_{k}-L\right| \geq \varepsilon\right\}\right|=0
$$

then the sequence $\left(x_{k}\right)$ is statistically convergent to $L$. 
The concepts of statistical limit superior and statistical limit inferior were introduced by Fridy and Orhan [6]. Let $k$ be a positive integer and $\mathrm{x}$ be a real number sequence. Define the sets $B_{x}$ and $A_{x}$ as

$$
B_{x}=\left\{b \in \mathbb{R}: \delta\left(\left\{n: x_{n}>b\right\}\right) \neq 0\right\}, \quad A_{x}=\left\{a \in \mathbb{R}: \delta\left(\left\{n: x_{n}<a\right\}\right) \neq 0\right\} .
$$

Then statistical limit superior and statistical limit inferior of $x$ is given by

$$
\begin{aligned}
& \text { st } \lim \sup x=\left\{\begin{array}{ccc}
\sup B_{x} & \text { if } & B_{x} \neq \emptyset, \\
-\infty & \text { if } & B_{x}=\emptyset .
\end{array}\right. \\
& \text { st- } \liminf x=\left\{\begin{array}{ccc}
\inf A_{x} & \text { if } & A_{x} \neq \emptyset, \\
+\infty & \text { if } & A_{x}=\emptyset .
\end{array}\right.
\end{aligned}
$$

Lemma 1.1 [6] If $\beta=$ st- $\lim \sup x$ is finite, then for every $\varepsilon>0$,

$$
\delta\left(\left\{k \in \mathbb{N}: x_{k}>\beta-\varepsilon\right\}\right) \neq 0 \text { and } \delta\left(\left\{k \in \mathbb{N}: x_{k}>\beta+\varepsilon\right\}\right)=0
$$

Conversely, if (1.1) holds for every, $\varepsilon>0$ then $\beta=\operatorname{st}-\lim \sup x$.

The dual statement for st- $\lim \inf x$ is as follows:

Lemma 1.2 [6] If $\alpha=\operatorname{st-lim} \inf x$ is finite, then for every $\varepsilon>0$,

$$
\delta\left(\left\{k \in \mathbb{N}: x_{k}<\alpha+\varepsilon\right\}\right) \neq 0 \text { and } \delta\left(\left\{k \in \mathbb{N}: x_{k}<\alpha-\varepsilon\right\}\right)=0
$$

Conversely, if (1.2) holds for every, $\varepsilon>0$ then $\alpha=\operatorname{st}-\lim \sup x$.

A point $\xi \in X$ is called a statistical limit point of a sequence $x=\left(x_{k}\right)$ if there is a set $K=k_{1}<k_{2}<k_{3}<\ldots$ with $\delta(K) \neq 0$ such that $x_{k_{n}} \rightarrow \xi$ as $\mathrm{n} \rightarrow \infty$. The set of all statistical limit points of a sequence $x$ will be denoted by $\Lambda_{x}$

A point $\xi \in X$ is called a statistical cluster point of $x=\left(x_{k}\right)$ if for any $\varepsilon>0$,

$$
\delta\left(\left\{k \in \mathbb{N}: d\left(x_{k}, \xi\right)<\varepsilon\right\}\right) \neq 0 .
$$

The set of all statistical cluster points of $\mathrm{x}$ will be denoted by $\Gamma_{x}$.

Let $L_{x}$ denote the set of all limit points $\xi$ (accumulation points) of the sequence $x$; i.e. $\xi \in L_{x}$ if there exists an infinite set $K=k_{1}<k_{2}<k_{3}<\ldots$ such that $x_{k_{n}} \rightarrow \xi$ as $\mathrm{n} \rightarrow \infty$.

Obviously we have $\Lambda_{x} \subseteq \Gamma_{x} \subseteq L_{x}$

In our study we will be interested much more on sequence of functions. Statistical convergence on sequence of functions is defined by Gokhan and Gungor [7].

Following definitions are statistical inner and outer limits on the concept of set convergence which is fundamental to define statistical hypo-limit by using sets. In this paper, we deal with Painleve-Kuratowski [9] convergence and actually its statistical version will be studied here which is defined by Sever and Talo [18]. In set convergence, following collections of subsets of $\mathbb{N}$ play an important role for defining statistical inner and outer limits on sequence of sets.

$$
\begin{gathered}
\mathcal{S}=\{N \subset \mathbb{N}: \delta(N)=1\}, \\
\mathcal{S}^{\#}=\{N \subset \mathbb{N}: \delta(N) \neq 0\} .
\end{gathered}
$$

Definition $1.3[18]$ Let $(X, d)$ be a metric space. Statistical inner and outer limit of a sequence $\left(A_{n}\right)$ of closed subsets of $X$ are defined as follows:

$$
\begin{aligned}
& s t-\liminf _{n} A_{n}=\left\{x \mid \forall V \in \mathcal{N}(x), \exists N \in \mathcal{S}, \forall n \in N: A_{n} \cap V \neq \emptyset\right\}, \\
& \text { st- } \liminf _{n} A_{n}=\left\{x \mid \forall \varepsilon>0, \exists N \in \mathcal{S}, \forall n \in N: A_{n} \cap B(x, \varepsilon) \neq \emptyset\right\} \\
& \text { st- } \limsup _{n} A_{n}=\left\{x \mid \forall V \in \mathcal{N}(x), \exists N \in \mathcal{S}^{\#}, \forall n \in N: A_{n} \cap V \neq \emptyset\right\} \\
& \text { st- } \limsup _{n} A_{n}=\left\{x \mid \forall \varepsilon>0, \exists N \in \mathcal{S}^{\#}, \forall n \in N: A_{n} \cap B(x, \varepsilon) \neq \emptyset\right\}
\end{aligned}
$$

Proposition 1.4 [18] Let $(X, d)$ be a metric space and $(A n)$ be a sequence of closed subsets of $X$. 
Then

$$
\text { st- } \liminf _{n} A_{n}=\left\{x \mid \exists N \in \mathcal{S}, \forall n \in N, \exists y_{n} \in A_{n}: \lim _{n} y_{n}=x\right\} .
$$

Proposition 1.5 [18] Let $(X, d)$ be a metric space and $(A n)$ be a sequence of closed subsets of $X$.

Then

$$
\text { st }-\limsup _{\mathrm{n}} A_{n}=\left\{x \mid \exists N \in S^{\#}, \forall n \in N, \exists y_{n} \in A_{n}: x \in \Gamma_{y_{n}}\right\}
$$

Let $\mathrm{f}$ be a function defined on $X$, the hypograph of $\mathrm{f}$ is the set hypo $(f)=\{(x, \alpha) \in X \times \mathbb{R} \mid \alpha \leq f(x)\}$ and its level set is defined by $\operatorname{lev}_{\geq \alpha} f=\{x \in X \mid f(x) \geq \alpha\}$.

Definition 1.6 [15] For any sequence (fn) of functions on $X$, the lower hypo-limit, $h$ - $\lim \inf _{n} f_{n}$, is the function having as its hypograph the outer limit of the sequence of sets hypo $f_{n}$ :

$$
\text { hypo }\left(h-\liminf _{n} f_{n}\right)=\liminf _{n}\left(\text { hypo }_{n}\right) .
$$

The upper hypo-limit, $h$ - $\lim \sup _{n} f_{n}$, is the function having as its hypograph the inner limit of the sequence of sets hypof $f_{n}$ :

$$
\text { hypo }\left(h-\limsup _{n} f_{n}\right)=\limsup _{n}\left(h y p o f_{n}\right) .
$$

When these two functions equal to each other, we have $h-\lim _{n} f_{n}=h$ - $\lim \inf _{n} f_{n}=h$ - $\lim \sup _{n} f_{n}$. Hence the functions $f_{n}$ are said to hypo-convergent to the function $f$. It is symbolized by $f_{n} \stackrel{h}{\rightarrow} f$ Moreover, the relation between set convergence and convergence of sequence of functions appears in the following equality.

$$
f_{n} \stackrel{h}{\rightarrow} f \Leftrightarrow \text { hypo }_{n} \rightarrow \operatorname{hypo}(f) .
$$

We also advise to look at $[3,4,14,16]$ for detailed information about new types of convergence of sequences of real valued functions and statistical convergence.

\section{Main Result}

In this part, statistical hypo-convergence is defined by the help of Kuratowski convergence on sets. The functions will be taken upper semicontinuous in order to use properties on closed sets since hypo-graphs of upper semicontinuous functions are closed. Set properties will give a new characterization of statistical hypo-convergence by using neighbourhoods of the point $x \in X$ in a metric space. Neighbourhoods will give other characterizations of statistical hypo-convergence by using sequences this time. Level sets which are important instruments in set theory are also included in our calculations for lower and upper statistical hypo-limits. Moreover, statistical hypo-convergence and statistical upper semicontinuity will be discussed at the end.

Definition 2.1 Let $(X, d)$ be a metric space and $\left(f_{n}\right)$ a sequence of upper semicontinuous functions defined from $X$ to $\overline{\mathbb{R}}$. The lower statistical hypo-limit, $h_{s t^{-}} \lim \inf _{n} f_{n}$ is defined by the help of the sequence of sets:

$$
\text { hypo }\left(h_{s t}-\liminf _{n} f_{n}\right)=s t-\liminf _{n}\left(\text { hypof }_{n}\right) .
$$

Similarly, the upper statistical hypo-limit $h_{s t}-\lim \sup _{n} f_{n}$ is defined:

$$
\text { hypo }\left(h_{s t}-\limsup _{n} f_{n}\right)=s t-\limsup _{n}\left(\text { hypo }_{n}\right) .
$$

When these two functions are equal, we get statistical hypo-limit function:

$$
f=h_{s t}-\lim _{n} f_{n}=h_{s t}-\limsup _{n} f_{n}=h_{s t}-\liminf _{n} f_{n} .
$$

As defined in above, it is obvious that $h_{s t^{-}} \lim \inf _{n} f_{n} \leq h_{s t^{-}} \lim \sup _{n} f_{n}$. 
Here we use statistical Painleve-Kuratowski convergence. Whenever $\left(f_{n}\right)$ is hypo-convergent to $f$ we can use the inclusion st- $\lim \sup _{n}\left(\right.$ hypof $\left._{n}\right) \subset$ hypof $\subset s t-\lim _{\inf }\left(\right.$ hypof $\left._{n}\right)$.

Moreover, following comparisons with hypo-limits are valid for every function $\mathrm{f}: X \rightarrow \overline{\mathbb{R}}$.

$$
h-\liminf _{n} f_{n} \leq h_{s t^{-}} \liminf _{n} f_{n}, \quad h-\limsup _{n} f_{n} \leq h_{s t^{-}} \limsup _{n} f_{n} .
$$

In the following example, the function is not hypo-convergent whereas it has statistical hypo-limit.

Example 2.2 Given a sequence $f_{n}: \mathbb{R} \rightarrow \overline{\mathbb{R}}$ defined as

$$
\begin{gathered}
f_{n}(x)=\left\{\begin{array}{c}
2 n x e^{-2 n^{2} x^{2}} \quad \text { if } n \text { is square, } \\
n x e^{-2 n^{2} x^{2}} \quad \text { if } n \text { is nonsquare. }
\end{array}\right. \\
h \text { - } \limsup _{n} f_{n}(x)=\left\{\begin{array}{ccc}
e^{-\frac{1}{2}} & \text { if } & x=0, \\
0 & \text { if } & x \neq 0,
\end{array}\right. \\
h \text { - } \liminf _{n} f_{n}(x)=\left\{\begin{array}{ccc}
\frac{1}{2} e^{-\frac{1}{2}} \text { if } & x=0, \\
0 & \text { if } & x \neq 0,
\end{array}\right. \\
h_{s t}-\lim _{n} f_{n}(x)=\left\{\begin{array}{ccc}
\frac{1}{2} e^{-\frac{1}{2}} \text { if } & x=0, \\
0 & \text { if } & x \neq 0 .
\end{array}\right.
\end{gathered}
$$

In this example, statistical hypo-convergence deals with supremum values of a sequence of functions even if ordinary hypo-convergence fails to find it. Hence, it makes statistical hypo-convergence more reliable for finding optimum values in stochastic optimization problems.

On the other hand, $\left(f_{n}\right)$ is statistically pointwise convergent to the function $f(x)=0$ which is different than hypo-limits. It shows us that statistical hypo-convergence is neither stronger nor weaker than statistical pointwise convergence. The obvious difference between these convergence types is obtaining supremums.

Now we will give characterizations of upper and lower statistical hypo-convergence using neighbourhoods. Before giving definitions, we need to prove following Lemma 2.3 and Lemma 2.4.

Lemma 2.3 Let $(X, d)$ be a metric space and $\left(f_{n}\right)$ a sequence of upper semicontinuous functions defined from $X$ to $\overline{\mathbb{R}}$, for every $x \in X$, define $g: X \rightarrow \overline{\mathbb{R}}$ by

$$
g(x)=\inf _{V \in \mathcal{N}(x)} s t-\limsup _{n} \sup _{y \in V} f_{n}(y) .
$$

Then st- $\lim \sup _{n}\left(\right.$ hypof $\left._{n}\right)=$ hypo $(g)$.

Proof: We should establish the epigraphical inclusions of the sets $s t$ - $\lim \sup _{n}\left(\right.$ hypof $\left._{n}\right) \subset$ hypo $(g)$ and hypo $(g) \subset s t$ - lim $\sup _{n}\left(\right.$ hypof $\left._{n}\right)$. For the first inclusion, let $(\mathrm{x}, \alpha) \in s t-\lim \sup _{n}\left(\right.$ hypof $\left._{n}\right)$ be arbitrary. Let $\mathrm{V}_{0} \in \mathcal{N}(x)$ and $\varepsilon>0$ be fixed. By definition of the statistical upper limit of sets, $\exists N \in \mathcal{S}^{\#}$ such that $\forall n \in N$ we have

$$
V_{0} \times(\infty, \alpha-\varepsilon) \bigcap \operatorname{hypof}_{n} \neq \emptyset \text {. }
$$

As a result,

$$
\delta\left(\left\{n \in \mathbb{N}: \sup _{y \in V_{0}} f_{n}(y)>\alpha-\varepsilon\right\}\right) \neq 0
$$


By Lemma 1.1 we have,

$$
s t-\limsup _{n} \sup _{y \in V_{0}} f_{n}(y) \geq \alpha \text {. }
$$

$V_{0}$ and $\varepsilon$ were arbitrary, we have $g(x)>\alpha$ and hence $(x, \alpha) \in$ hypo $(g)$.

For the second inclusion let $(x, \alpha) \in \operatorname{hypo}(g)$, for all $\mathrm{V}_{0} \in \mathcal{N}(x)$ and for all $\varepsilon>0$ we have,

$$
\alpha \leq g(x) \leq s t-\lim \sup _{n} \sup _{y \in V_{0}} f_{n}(y) .
$$

Again by Lemma 1.1, we get $\delta\left(\left\{n \in \mathbb{N}\right.\right.$ : $\left.\left.\sup _{y \in V_{0}} f_{n}(y)>\alpha-\varepsilon\right\}\right) \neq 0$. It means, $\exists N \in \mathcal{S}^{\#}$ such that $\forall n \in N$

$$
V_{0} \times(\infty, \alpha-\varepsilon) \bigcap \text { hypof }_{n} \neq \emptyset .
$$

Hence $(\mathrm{x}, \alpha) \in$ st- $\lim \sup _{n}\left(\right.$ hypof $\left._{n}\right)$.

Lemma 2.4 Let $(X, d)$ be a metric space and $\left(f_{n}\right)$ a sequence of upper semicontinuous functions defined from $X$ to $\overline{\mathbb{R}}$, for every $x \in X$, define $h: X \rightarrow \overline{\mathbb{R}}$ by

$$
h(x)=\inf _{V \in \mathcal{N}(x)} s t-\liminf _{n} \sup _{y \in V} f_{n}(y) .
$$

Then st $\lim \inf _{n}\left(\operatorname{hypof}_{n}\right)=$ hypo $(h)$

Proof: We want to show st- $\lim \sup _{n}\left(h_{y p o f_{n}}\right) \subset$ hypo $(h)$ and hypo $(h) \subset s t-\lim \inf _{n}\left(h_{y p o f}\right)$. For the first inclusion, let $(x, \alpha) \in s t-\lim \sup _{n}\left(h_{y p o f}\right)$ be arbitrary. Let $\mathrm{V}_{0} \in \mathcal{N}(x)$ and $\varepsilon>0$ be fixed. By definition of the statistical lower limit of sets, $\exists N \in \mathcal{S}$ such that $\forall n \in N$ we have

$$
V_{0} \times(\infty, \alpha-\varepsilon) \bigcap \operatorname{hypof}_{n} \neq \emptyset .
$$

As a result,

$$
\delta\left(\left\{n \in \mathbb{N}: \sup _{y \in V_{0}} f_{n}(y)<\alpha-\varepsilon\right\}\right)=0
$$

By Lemma 1.1 we have,

$$
\text { st- } \liminf _{n} \sup _{y \in V_{0}} f_{n}(y) \geq \alpha .
$$

$V_{0}$ and $\varepsilon$ was arbitrary, we have $h(x) \geq \alpha$ and hence $(x, \alpha) \in$ hypo $(h)$.

For the second inclusion, fix $(x, \alpha) \in$ hypo $(h)$. Given $\mathrm{V}_{0} \in \mathcal{N}(x)$ and $\varepsilon>0, \exists N \in \mathcal{S}$ such that $\forall n \in N$ we have

$$
\text { st- } \liminf _{n} \sup _{y \in V_{0}} f_{n}(y) \geq h(x)>\alpha-\varepsilon
$$

and it equals to the following equality

$$
\delta\left(\left\{n \in \mathbb{N}: \sup _{y \in V_{0}} f_{n}(y)>\alpha-\varepsilon\right\}\right)=1 .
$$

Hence,

$$
\delta\left(\left\{n \in \mathbb{N}: V_{0} \times(\infty, \alpha-\varepsilon) \bigcap \text { hypo }_{n} \neq \emptyset\right\}\right)=1 .
$$

We conclude that 


$$
\delta\left(\left\{n \in \mathbb{N}: V_{0} \times(\alpha-\varepsilon, \alpha+\varepsilon) \bigcap \text { hypof }_{n} \neq \emptyset\right\}\right)=1 .
$$

It gives $(x, \alpha) \in s t$ - $\lim \inf _{n}\left(\right.$ hypof $\left._{n}\right)$ and concludes the proof.

Next definition gives us a characterization of hypo-limits with the help of Lemma 2.3 and Lemma 2.4.

Definition 2.5 Let $(X, d)$ be a metric space and $\left(f_{n}\right)$ a sequence of lower semicontinuous functions from $X$ into $\overline{\mathbb{R}}$, for every $x \in X$, lower and upper statistical epi-limit functions are defined by

$$
\begin{aligned}
& \left(h_{s t}-\liminf _{n} f_{n}\right)(x)=\inf _{V \in \mathcal{N}(x)} s t-\liminf _{n} \sup _{y \in V} f_{n}(y) \\
& \left(h_{s t}-\limsup _{n} f_{n}\right)(x)=\inf _{V \in \mathcal{N}(x)} s t-\lim \sup _{n} \sup _{y \in V} f_{n}(y)
\end{aligned}
$$

If there exists a function $f: X \rightarrow \overline{\mathbb{R}}$ such that $h_{s t^{-}} \lim \inf _{n} f_{n}=h_{s t^{-}} \lim \sup _{n} f_{n}=f$, then we write $f=h_{s t^{-}} \lim _{n} f_{n}$ and we say that $\left(f_{n}\right)$ is $h_{s t}$-convergent to $f$ on $X$.

Lemma 2.6 Let $x=\left(x_{n}\right)$ be a real sequence. Then

$$
\begin{aligned}
& s t-\liminf _{n \rightarrow \infty} x_{n}=\inf _{N \in \mathcal{S} \#} \sup _{n \in N} x_{n}=\sup _{N \in \mathcal{S}} \inf _{n \in N} x_{n} \\
& s t-\limsup _{n \rightarrow \infty} x_{n}=\sup _{N \in \mathcal{S}^{\#}} \inf _{n \in N} x_{n}=\inf _{N \in \mathcal{S}} \sup _{n \in N} x_{n}
\end{aligned}
$$

By lemma 2.6, the statistical hypo-limit infimum can be expressed as follows:

$$
\left(h_{s t^{-}} \liminf _{n} f_{n}\right)(x)=\inf _{V \in \mathcal{N}(x)} \inf _{N \in \mathcal{S}} \sup _{n \in N} \sup _{y \in V} f_{n}(y)=\inf _{V \in \mathcal{N}(x)} \sup _{N \in \mathcal{S}} \inf _{n \in N} \sup _{y \in V} f_{n}(y) .
$$

Similarly, the statistical hypo-limit supremum can be expressed as follows:

$$
\left(h_{s t^{-}} \lim \sup _{n} f_{n}\right)(x)=\inf _{V \in \mathcal{N}(x)} \sup _{N \in \mathcal{S}_{\#}^{\#}} \inf _{n \in N} \sup _{y \in V} f_{n}(y)=\inf _{V \in \mathcal{N}(x)} \inf _{N \in \mathcal{S}} \sup _{n \in N} \sup _{y \in V} f_{n}(y)
$$

Proposition 2.7 In a metric space $(X, d)$ for every $x \in X$, the following inequalities hold:

$$
\left(h_{s t}-\liminf _{n} f_{n}\right)(x) \geq s t-\liminf _{n} f_{n}(x), \quad\left(h_{s t}-\limsup _{n} f_{n}\right)(x) \geq s t-\limsup _{n} f_{n}(x) .
$$

Proof: $\forall x \in X, \forall V \in \mathcal{N}(x), \exists N \in \mathcal{S}$ such that $\forall n \in N$ we have

$$
\sup _{y \in V} f_{n}(y) \geq f_{n}(x), \quad \sup _{y \in V} f_{n}(y) \geq f_{n}(x) .
$$

Since by the choice of our index set $(n \in N)$, we get the following inequalities,

$$
s t-\liminf _{n} \sup _{y \in V} f_{n}(y) \geq s t-\liminf _{n} f_{n}(x), \quad s t-\limsup _{n} \sup _{y \in V} f_{n}(y) \geq s t-\limsup _{n} f_{n}(x) .
$$

After taking the infimum over all $V \in \mathcal{N}(x)$ we get the desired conclusion.

Theorem 2.8 Let $(X, d)$ be a metric space and let $\left(f_{n}\right)$ be a sequence of upper semicontinuous functions. Suppose that for each $\alpha \in \mathbb{R}, \exists\left(\alpha_{\mathrm{n}}\right)$ of reals statistically convergent to $\alpha$ with lev $v_{\geq \alpha} f=s t-\lim _{n}\left(l e v_{\geq \alpha_{n}} f_{n}\right)$, then $f=h_{s t}-\lim _{n} f_{n}$. 
Proof: The condition $l e v_{\geq \alpha} f \subset s t-\lim _{\inf _{n}}\left(\operatorname{lev}_{\geq \alpha_{n}} f_{n}\right)$ valid for each $\alpha \in \mathbb{R}$ and for some sequence $\alpha_{\mathrm{n}} \stackrel{\text { st }}{\rightarrow} \alpha$. Let $(x, \alpha) \in$ hypo $(f)$ then there exists a sequence $\alpha_{\mathrm{n}}$ statistically convergent to $\alpha$ such that $\operatorname{lev}_{\geq \alpha} f \subset s t-\lim \inf _{n}\left(\operatorname{lev} v_{\geq \alpha_{n}} f_{n}\right)$. Hence $\mathrm{x}$ $\in s t-\lim \inf _{n}\left(l e v_{\geq \alpha_{n}} f_{n}\right)$. It means there exists a sequence $\left(x_{n}\right)$ statistically convergent to $x$ such that $\mathrm{x}_{\mathrm{n}} \in\left(l e v_{\geq \alpha_{n}} f_{n}\right)$. Finally we get $\left(x_{\mathrm{n}}, \alpha_{\mathrm{n}}\right) \stackrel{\text { st }}{\rightarrow}(x, \alpha)$ and $(x, \alpha) \in$ st $\lim _{\inf _{n}}$ hypof $f_{n}$.

In order to get st- $\lim \sup _{\mathrm{n}}$ hypof $_{n} \subset$ hypo $(f)$, suppose to the contrary that $(\mathrm{x}, \beta) \in$ st- $\lim \sup _{n}$ hypof $f_{n}$ but that $(\mathrm{x}, \beta) \notin$ hypo $(f)$. Then $\beta>f(x)$. We can find $N \in \mathcal{S}^{\#}$ such that $\forall n \in N\left(x_{n}, \beta_{n}\right) \in$ hypof $_{n}$ such that $(\mathrm{x}, \beta) \in \Gamma\left(x_{n}, \beta_{n}\right)$. Choose a scalar $\alpha$ between $\beta$ and $f(x)$ and let $\left(\alpha_{n}\right)$ be a sequence statistically convergent to $\alpha$ for which $\operatorname{lev}_{\geq \alpha} f \supset s t$ - lim $\sup _{n}\left(l e v_{\geq \alpha_{n}} f_{n}\right)$. We have $\delta\left(n: \beta_{n}>\alpha_{n}\right) \neq 0$ and $\left(x_{n}, \beta_{n}\right) \in$ hypof $_{n} . \exists N \in \mathcal{S}^{\#}, \forall n \in N, x_{n} \in l e v_{\geq \alpha_{n}} f_{n}$ which means $x \in$ st- $\lim \sup l e v_{\geq \alpha_{n}} f_{n}$. By the inclusion st-lim sup $\sup _{n} l e \alpha_{n} f_{n} \subset \operatorname{le} v_{\geq \alpha} f$, we get $x \in \operatorname{lev}_{\geq \alpha} f$ and $f(x) \geq \alpha$ which is a contradiction.

Theorem 2.9 Let $\left(f_{n}\right)$ and $f$ be functions from $X$ to $\overline{\mathbb{R}}$ with $f$ upper semicontinuous. hypo $(f) \subset$ st- $\lim \inf _{n}$ hypof $f_{n}$ if and only iffor every open set $\mathcal{O}$ with hypo $(f) \cap \mathcal{O} \neq \emptyset$; there exists $N \in \mathcal{S}$ such that hypo $f_{n} \cap \mathcal{O} \neq \emptyset$ for all $n \in N$.

Proof: Necessity comes directly from (1.3). To illustrate sufficiency, suppose that there exists $x$ in hypo(f) but not in st$\lim \inf _{n}$ hypof $_{n}$. But then by (1.3), there exists an open neighborhood $V$ of $x$ such that for every $N \in \mathcal{S}$ there exists $n \in N$ with $V \cap$ hypof $_{n}=\emptyset$; and also $V \cap$ hypo $(f) \neq \emptyset$. This is the negation of the condition on the right.

Theorem 2.10 Let $\left(f_{n}\right)$ and f be functions from $X$ to $\overline{\mathbb{R}}$ with $f$ upper semicontinuous. hypo $(f) \supset$ st- $\lim \sup _{n}$ hypof f $_{n}$ and only if for every compact set $C$ with hypo $(f) \cap C=\emptyset$ there exists $N \in \mathcal{S}$ such that hypof $f_{n} \cap C=\emptyset$ for all $n \in N$.

Proof: Let hypo(f) $\supset s t$ - $\lim \sup _{n}$ hypof $_{n}$ and let there exists a compact set $C$ with hypo $(f) \cap C=\emptyset$, such that for any $N \in$ $\mathcal{S}$ one has hypof ${ }_{n} \cap C \neq \varnothing$ for some $n \in N$. But then there exists $N \in \mathcal{S}^{\#}$ and a statistically convergent sequence $x_{n} \in$ hypof $_{n}$ for $n \in N$ whose statistical limit not in hypo $(f)$, this is a contradiction. On the other hand, if there exists $x$ in st$\lim \sup _{n}$ hypof $_{n}$ which is not in hypo(f) then from (1.6), a ball $\mathrm{B}(\mathrm{x}, \varepsilon)$ with sufficiently small radius $\varepsilon$ does not meet $h y$ $p o(f)$ yet meets $h_{y p o f}$ for infinitely many $n$; this contradicts the condition on the right.

Corollary 2.11 Let $\left(f_{n}\right)$ and f be functions from $X$ to $\overline{\mathbb{R}}$ with $f$ upper semicontinuous. hypo $(f) \subset$ st- $\lim _{\inf _{n}}$ hypof $_{n}$ if and only if whenever hypo $(f) \cap B(x, \varepsilon) \neq \varnothing$ for a ball $B(x, \varepsilon)$, there exists $N \in \mathcal{S}$ such that hypof $\cap \cap B(x, \varepsilon) \neq \emptyset$ for all $n \in N$.

Proof: It is clear from Theorem 2.9.

Corollary 2.12 Let $\left(f_{n}\right)$ and f be functions from $X$ to $\overline{\mathbb{R}}$ with $f$ upper semicontinuous. hypo $(f) \subset$ st- $\lim _{\sup _{n}}$ hypof $f_{n}$ if and only if whenever hypo(f) $\cap \bar{B}(x, \varepsilon)=\emptyset$ for a ball $\bar{B}(x, \varepsilon)$, there exists $N \in \mathcal{S}$ such that hypof ${ }_{n} \cap \bar{B}(x, \varepsilon)=\emptyset$ for all $n \in N$.

Proof: It is clear from Theorem 2.10.

Theorem 2.13 Let $\left(f_{n}\right)$ and $f$ be functions from $X$ to $\overline{\mathbb{R}}$ with $f$ upper semicontinuous. $h_{s t} \lim _{\inf } f_{n} \leq f$ if and only if st$\lim \sup _{n}\left(\sup _{C} f_{n}\right) \leq \sup _{C} f$ for every compact set $\mathrm{C} \subset \mathrm{X}$.

Proof: For necessity, assume that $h_{s t^{-}} \lim \inf _{n} f_{n} \leq f$ which means $s t-\lim \sup _{\mathrm{n}}\left(\right.$ hypof $\left.f_{n}\right) \subset$ hypo $(f)$. Let us take any compact set $C \subset X$ and a value $\alpha \in \overline{\mathbb{R}}$ such that $\sup _{C} f<\alpha$. Then the compact set $(C, \alpha)$ in $X \times \overline{\mathbb{R}}$ does not meet hypo(f). Hence by Theorem 2.10 there exists $N \in \mathcal{S}$ such that for all $n \in N,(\mathrm{C}, \alpha)$ does not meet hypof $f_{n}$ either. This implies $\sup _{C} f_{n}$ $\leq \alpha$ and because of the choice of our index set we obtain $s t$ - $\lim \sup _{n}\left(\sup _{C} f_{n}\right) \leq \sup _{C} f$.

For sufficiency, let $B^{+}((x, \alpha), \varepsilon)$ be a cylinder does not meet hypo $(f)$ defined as,

$$
B^{+}((x, \alpha), \varepsilon):=\bar{B}(x, \varepsilon) \times[\alpha-\varepsilon, \alpha+\varepsilon] .
$$

Given $f$ is upper semicontinuous, so that hypo $(f)$ is closed and $\sup _{\bar{B}(x, \varepsilon)} f<\alpha-\varepsilon$. Since the ball 
$\bar{B}(x, \varepsilon)$ is a compact set in $X$, we know by assumption that $s t-\lim \inf _{n}\left(\sup _{\bar{B}(x, \varepsilon)} f\right) \leq \alpha-\varepsilon$. Therefore

there exists $N \in \mathcal{S}$ such that $\sup _{\bar{B}(x, \varepsilon)} f_{n}<\alpha-\varepsilon$ for all $n \in N$. Then the cylinder $B^{+}((x, \alpha), \varepsilon)$ does not meet hypof $f_{n}$ for any $n \in N$. By Corollary 2.12 we have st- $\lim \sup _{n}\left(\right.$ hypof $\left._{n}\right) \subset$ hypo $(f)$ and we obtain $h_{s t}-\lim \inf _{n} f_{n} \leq f$.

Theorem 2.14 Let $\left(f_{n}\right)$ and $f$ be functions from $X$ to $\overline{\mathbb{R}}$ with $f$ upper semicontinuous. $h_{s t} \lim \sup _{n} f_{n} \geq f$ if and only if st- $\left.\liminf \operatorname{inp}_{n} f_{n}\right) \geq \sup _{\mathcal{O}} f$ for every open set $\mathcal{O} \subset X$.

Proof: The proof is similar to the previous one.

Definition 2.15 The sequence $\left(f_{n}\right)$ is called statistically equi upper semicontinuous at a point $x$ if and only if for all $\varepsilon>0$ there exists $\delta>0$ and $N \subset \mathcal{S}$ such that for all $y \in B(x, \delta)$ we have,

$$
f_{n}(x)+\varepsilon>f_{n}(y)
$$

for each $n \in N$.

Next theorem gives the basic condition that statistical convergence and statistical hypo-convergence coincide.

Theorem $2.16\left(f_{n}\right)$ and $f$ are functions from $X$ to $\mathbb{R}$, let $\left(f_{n}\right)$ be statistically equi upper semi-continuous at $x$. $\left(f_{n}\right)$ is statistically hypo-convergent to $f$ at $x$ if and only if $\left(f_{n}\right)$ is statistically convergent to $f$ at $x$.

Proof: Assuming $\left(f_{n}\right)$ is statistically upper semicontinuous at $x$, we have that for all $\varepsilon>0$, there

exists $V \in \mathcal{N}(x)$, and $N \in \mathcal{S}$ such that

$$
f_{n}(x)+\varepsilon>\sup _{y \in V} f_{n}(y)
$$

for all $n \in N$. This implies

$$
\text { st- } \liminf _{n} f_{n}(x)+\varepsilon \geq \inf _{V \in \mathcal{N}(x)} \text { st- } \liminf _{n} \sup _{y \in V} f_{n}(y)=h_{s t^{-}} \liminf _{n} f_{n}(x)
$$

for every $\varepsilon>0$. Combining with Proposition 2.7 we get

$$
\text { st- } \liminf _{n} f_{n}(x)=\inf _{V \in \mathcal{N}(x)} \text { st- } \liminf _{n} \sup _{y \in V} f_{n}(y)
$$

which means,

$$
\text { st- } \liminf _{n} f_{n}(x)=h_{s t^{-}} \liminf _{n} f_{n}(x) .
$$

In similar way, we get $s t-\lim \sup _{n} f_{\mathrm{n}}(x)=h_{s t^{-}} \lim \sup _{n} f_{\mathrm{n}}(x)$ and finally we reach the desired equality as follows

$$
\text { st- } \lim _{n} f_{n}(x)=h_{s t^{-}} \lim _{n} f_{n}(x) \text {. }
$$

\section{Conclusion}

The results in this paper which we derived from statistical Kuratowski limits will be used for our further studies related with maximizers of statistical lower and upper hypo-limit functions. Since, statistical hypo-convergence of sequence of functions is important for maximization of stochastic optimization and variational problems.

\section{References}

[1] Anastassiou, A. G., Duman O.: Towards Intelligent Modeling: Statistical Approximation Theory, vol.14. Berlin (2011)

[2] Attouch, H.: Convergence de fonctions convexes, de sous-differentiels et semi-groupes. Comptes Rendus de 1Academie des 
Sciences de Paris. 284 539-542 (1977)

[3] Das, R., Papanastassiou, N.: Some types of convergence of sequences of real valued functions. Real Anal. Exchange 28 (2) $1-16(2002 / 2003)$

[4] Di Maio, G., Kocinac, Lj. D. R.: Statistical convergence in topology. Topology Appl. 156 28-45 (2008)

[5] Fast, H.: Sur la convergence statistique. Colloq. Math. 2 241-244 (1951)

[6] Fridy, J. A., Orhan, C.: Statistical limit superior and limit inferior. Proc. Amer. Math. Soc.125 3625-3631 (1997)

[7] Gokhan, A., Gungor, M.: On pointwise statistical convergence. Indian J. pure appl. Math.33 (9) 1379-1384 (2002)

[8] Joly, J.-L.: Une famille de topologies sur lensemble des fonctions convexes pour lesquelles la polarite est bicontinue. Journal de Mathematiques Pures et Appliquees. 52 421-441 (1973)

[9] Kuratowski, C.: Topologie, vol.I. PWN, Warszawa (1958)

[10] Maso, G. D.: An introduction to Г-convergence, vol.8. Boston (1993)

[11] McLinden, L., Bergstrom, R.: Preservation of convergence of sets and functions infinite dimensions. Trans. Amer. Math. Soc 268 127-142 (1981)

[12] Mosco, U.: Convergence of convex sets and of solutions of variational inequalities. Adv. Math.3 510-585 (1969)

[13] Niven, I., Zuckerman, H. S.: An Introduction to the Theory of Numbers. New York (1980)

[14] Papanastassiou, N.: On a new type of convergence of sequences of functions. Atti Sem. Mat. Fis. Univ. Modena 50 493-506 (2002)

[15] Rockafellar, R.T., Wets, R.J-B.: Variational Analysis. (2009)

[16] Salat, T.: On statistically convergent sequences of real numbers. Math. Slovaca 30 139-150(1980)

[17] Salinetti, G., Wets, R.J-B.: On the relation between two types of convergence for convex functions. J. Math. Anal. Appl. $60211-226(1977)$

[18] Talo, O., Sever, Y., Basar, F.: On statistically convergent sequences of closed sets. Filomat. 30 (6) 1497-1509 (2016)

[19] Wets, R.J-B.: Convergence of convex functions, variational inequalities and convex optimization problems. New York (1980)

[20] Wijsman, R. A.: Convergence of sequences of convex sets, cones and functions. Bull. Amer. Math. Soc. 70 186-188 (1964)

[21] Wijsman, R. A.: Convergence of sequences of convex sets, cones and functions II. Trans. Amer. Math. Soc. 123 32-45 (1966) 